\title{
A Promising Mouse Model of Graves' Orbitopathy Induced by Adenovirus Expressing Thyrotropin Receptor A Subunit
}

\author{
Meng Zhang, Xi Ding, Li-Ping Wu, Ming-Qian He, Zi-Yi Chen, Bing-Yin Shi, and Yue Wang
}

Background: Graves' orbitopathy (GO) is the most common and serious manifestation of Graves' disease (GD). It is characterized by orbital inflammation and tissue remodeling. Although several GO models have been reported, most lack a full assessment or mechanistic evaluation. Here, we established a promising mouse model mimicking many aspects of human GO with a frequency of $70 \%$ and characterized the key role of T cells in the progression of GO.

Methods: An adenovirus expressing the human thyrotropin (TSH) receptor A subunit (Ad-TSHRA) was injected in the muscles of female BALB/C mice nine times to induce GO. At predetermined time points, histological examinations of retrobulbar tissues and thyroid glands were performed to dynamically monitor changes; serum autoantibodies and total thyroxine levels were examined to evaluate thyroid function. Flow cytometry of CD4+ T cell subgroups and RNA sequencing (RNA-Seq) of splenocytes were also performed to explore the underlying mechanism.

Results: After nine injections, 7 of 10 mice challenged with Ad-TSHRA developed the orbital changes associated with GO. Seven mice manifested retrobulbar fibrosis, and four mice showed adipogenesis. Exophthalmia, conjunctival redness, and orbital lymphocyte infiltration were also observed in a subset of mice. The orbitopathy was first detected after seven injections and followed the hyperplastic change observed in thyroids after four injections. Flow cytometry revealed increased proportions of Th1 cells and decreased proportions of Th2 cells and regulatory $\mathrm{T}$ (Treg) cells in the splenocytes of GO mice. This change in CD4+ T cell subgroups was confirmed by orbital immunohistochemical staining. Genes involved in T cell receptor signaling, proliferation, adhesion, inflammation, and cytotoxicity were upregulated in GO mice according to the RNA-Seq; a trend of upregulation of these GO-specific genes was observed in mice with hyperthyroidism without orbitopathy after four injections.

Conclusions: A GO mouse model was successfully established by administering nine injections of Ad-TSHRA. The model was achieved with a frequency of $70 \%$ and revealed the importance of T cell immunity. A potential time window from Graves' hyperthyroidism to GO was presented for the first time. Therefore, this model could be used to study the pathogenesis and novel treatments for GO.

Keywords: Graves' orbitopathy, retrobulbar fibrosis, retrobulbar adipogenesis, TSHR, CD4+ T cell subgroups

\section{Introduction}

G RAVES’ DISEASE (GD) IS an organ-specific autoimmune disease and the most common cause of hyperthyroidism in adults (1). Approximately $20-50 \%$ of GD patients suffer from Graves' orbitopathy (GO), which manifests as exophthalmos, swelling, diplopia, and decreased visual acuity. As an autoimmune-mediated inflammatory disorder, GO is characterized by adipogenesis, hyaluronic acid synthesis, and an enlargement of the extraocular muscles; however, the pathogenesis remains unclear (2-4). As the therapeutic options for GO are currently quite limited and unsatisfactory, there is a pressing need to develop novel strategies for the management of GO $(2,5)$.

Animal models have contributed to a better understanding of disease pathogenesis and the development of novel treatments. Since the last century, scientists have been working to establish an ideal animal model of GO. In the 1990s, the

Department of Endocrinology, The First Affiliated Hospital of Xi'an Jiaotong University, Xi'an, China. 
transfer of human thyrotropin (TSH) receptor (TSHR)primed $T$ cells to naive syngeneic $\mathrm{BALB} / \mathrm{c}$ mice resulted in orbitopathy, as manifested by the disorganization of muscle fibers, accumulation of adipose tissue, and infiltration of immune cells, accompanied by severe thyroiditis (6). Genetic immunization with TSHR cDNA has been a valid method to induce the production of serum autoantibodies (TRAbs) and hyperthyroidism in both mice and monkeys (7-9). Recently, plasmid transfection through electroporation reproduced several orbital features of GO, including fibrosis, adipogenesis, glycosaminoglycan deposition, and infiltration by CD3+ $\mathrm{T}$ lymphocytes and F4/80+ macrophages $(10,11)$. In another study, hyperthyroidism and orbitopathy, as evidenced by adipogenesis and hyaluronic acid deposition, were induced (12). However, differences in thyroid function were observed in the animal models constructed using the same technology in different locations $(11,13)$. More recently, a study involving nine injections of an adenovirus expressing the human TSHR A subunit (Ad-TSHRA), which led to long-term hyperthyroidism and orbital fibrosis, was reported, and no other orbital changes were mentioned (14-17). Thus, additional studies are needed to develop a GO model for use in practice.

In the clinic, orbital symptoms often occur after the onset of hyperthyroidism (predominantly within 18 months), even though they have been shown to evolve from a single systemic process $(2,18)$. The progression from Graves' hyperthyroidism $(\mathrm{GH})$ to $\mathrm{GO}$ has been described as a "double whammy," recapitulating thyroid dysfunction and the subsequent development of orbital complications over time (19). Reproducing this progression in an animal model will help researchers explore the relationship between $\mathrm{GH}$ and $\mathrm{GO}$, which has not been reported in previous studies.

In this study, we established a mouse model of GO by administering nine injections of Ad-TSHRA and revealed a time window from $\mathrm{GH}$ to GO for the first time. This model mimicked many aspects of human GO and achieved a frequency of up to $70 \%$. The progression from GH to GO was highlighted by a series of changes in $\mathrm{T}$ cell immunity. This promising model might accelerate studies of the pathogenesis and novel treatments for GO.

\section{Materials and Methods}

\section{Animal study}

Six-week-old female BALB/C mice were purchased from Charles River Laboratory (Beijing, China). They were housed in cages with filter-top lids and maintained at $18-22^{\circ} \mathrm{C}$ on a 12-hour light-dark cycle. All animal care procedures and treatments were performed according to the Guide for the
Care and Use of Laboratory Animals and were approved by the local authorities at Jiaotong University School of Medicine, Xi'an, China.

The animals were randomly divided into two groups based on body weight. Twenty-two mice received Ad-TSHRA, and eight mice received the same dose of an adenovirus expressing green fluorescent protein (Ad-EGFP). A prolonged immunization protocol was followed with three injections administered at a 3-week interval as the initiation phase and six additional booster injections at a 4-week interval as the maintenance phase (14). A volume of $25 \mu \mathrm{L}$ of the adenovirus was injected into the left and right femoral muscles each time $\left(10^{8} \mathrm{PFU}\right)$. After nine injections (at the 34th week after the first injection), 10 mice challenged with Ad-TSHRA and 8 mice challenged with Ad-EGFP were euthanized. After four and seven injections (at the 11th and 23rd week, respectively, after the first injection), six randomly selected mice challenged with Ad-TSHRA were euthanized for dynamic observations (Fig. 1A).

\section{Histological examinations of orbital tissues}

The orbital tissues were completely dissected, fixed, decalcified, embedded, and sliced into four coronal sections at $1-\mathrm{mm}$ intervals across the entire orbit. Then, the sections $(5 \mu \mathrm{m})$ were stained with hematoxylin-eosin (HE) or Masson's trichrome (14). Images were captured using a $4 \times$ objective lens (Olympus BX53, Japan).

The fibrotic areas in the retrobulbar adipose tissue and retrobulbar muscle were stained blue with Masson's trichrome. An analysis of the blue pixels in digital images was performed using the luminescence tool in Adobe Photoshop version CS5 (Adobe Systems, San Jose, CA) (20). The total fibrosis volume $\left(\mathrm{mm}^{3}\right)$ in the retrobulbar region of each orbit was calculated without the eyelids and eyeballs. Quantitative analysis of HE-stained retrobulbar adipose tissues was also performed in Adobe Photoshop, with the optic nerve serving as an anatomical landmark.

Most of the pathological features were confirmed by quantitative evaluations. In contrast to a previous study (15), only the fibrosis in the retrobulbar region was calculated rather than the fibrosis in the entire orbit because exophthalmos stems from the increased pressure within the bony cavity of the eye (2).

\section{Measurements of serum TRAbs and thyroid function}

TRAbs were measured using a TSH-binding inhibition assay according to the manufacturer's protocol (Medipan $\mathrm{GmbH}$, Berlin, Germany) (21). Total thyroxine (TT4) levels were measured with commercial kits as previously described (21).

FIG. 1. (A) Animal study schema. (B) OB, orbital bone; ON, optic nerve. (a) Masson-stained section. The arrow indicates retrobulbar fibrosis. (b) Total fibrosis volume in the retrobulbar space of each orbit of the Ad-EGFP- and Ad-TSHRA-challenged mice at week 34. (c) Total fibrosis volume at different times, with the mean \pm 2 SD of the Ad-EGFP-challenged mice as the normal range. (C) (a) HE-stained section. The arrow indicates retrobulbar adipose tissue. (b) Adipose tissue area in the retrobulbar space of each orbit of the Ad-EGFP- and Ad-TSHRA-challenged mice at week 34. (c) Adipose tissue area at different times, with the mean \pm 2 SD of the Ad-EGFP-challenged mice as the normal range. (D) Frontal and lateral appearance of the head regions. (a, c) AdEGFP-challenged mice. (b, d) Ad-TSHRA-challenged mice at week 34. Red arrows indicate eyes with exophthalmia and conjunctiva redness, and blue arrows indicate eyes with eyelid broadening. (E) Lymphocyte infiltration in different parts of the orbit. (a) Muscle bundles, (b) perioptic nerve, (c) connective tissue, and (d) adipose tissue. $* p<0.05$. Ad-EGFP, adenovirus expressing green fluorescent protein; Ad-TSHRA, adenovirus thyrotropin receptor A subunit; GH, Graves' hyperthyroidism; SD, standard deviation. 
A Ad immunization

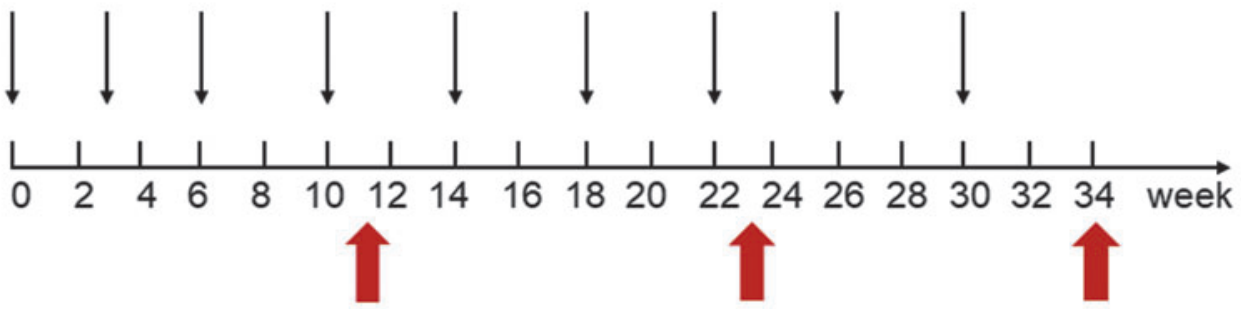

B
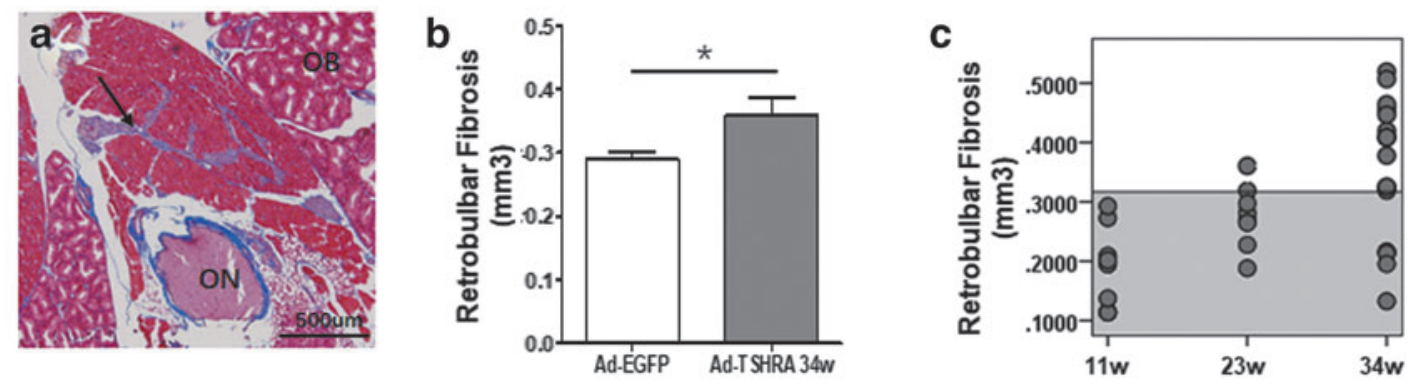

C
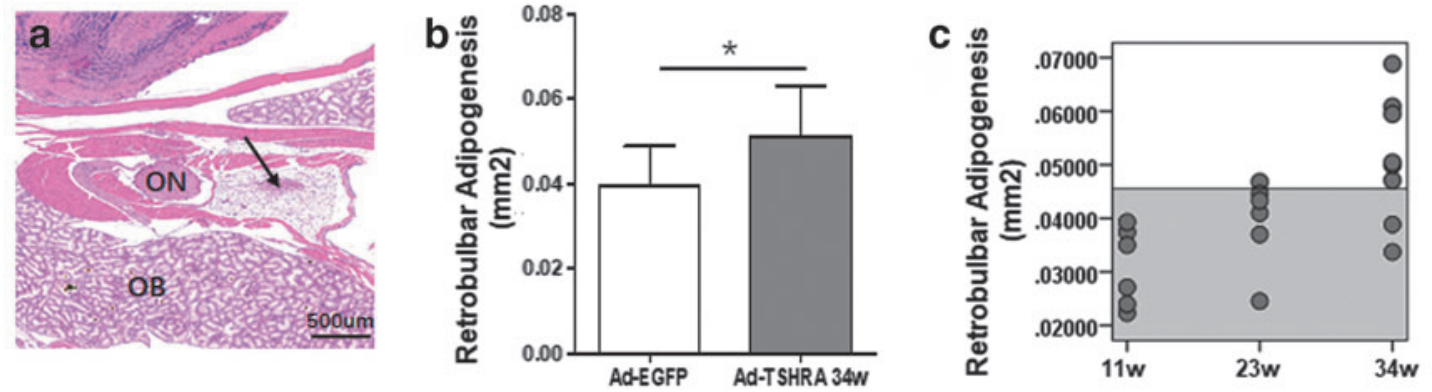

D
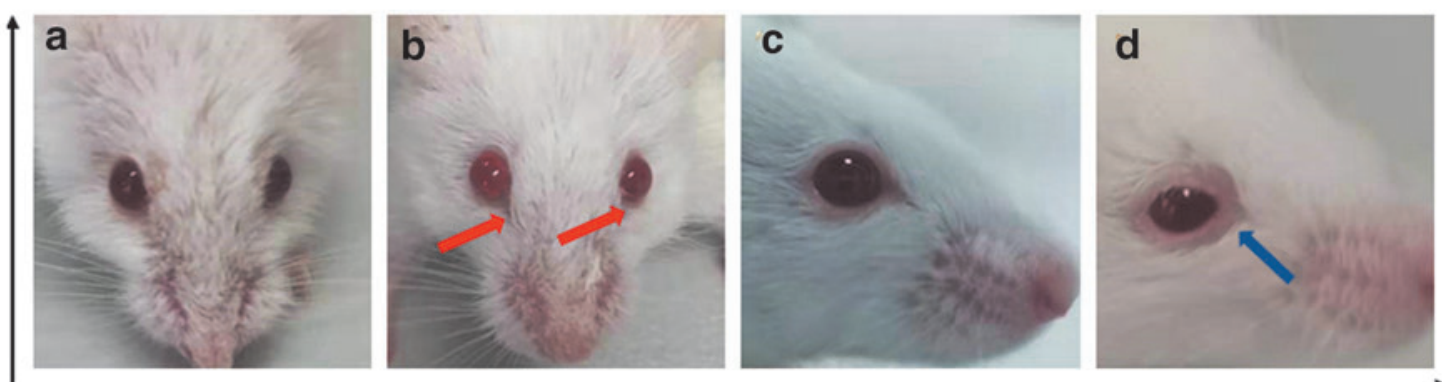

E

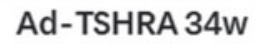

Ad-EGFP

Ad-TSHRA 34w
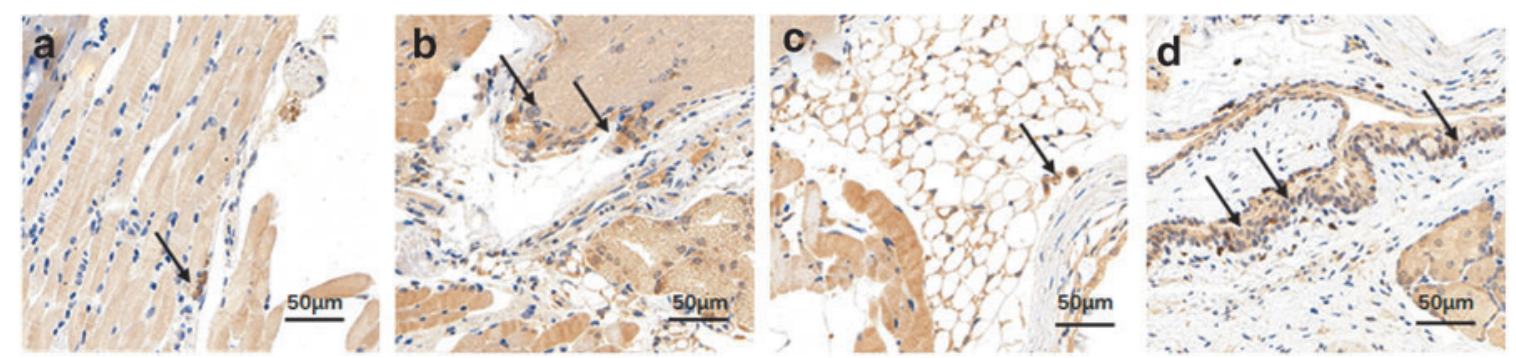


\section{Histological examinations of thyroid glands}

After euthanasia, cross-sectional incisions were generated above the larynx and below the seventh cartilage ring, and the thyroid glands were removed. Tissue blocks were immersed in $4 \%$ neutral paraformaldehyde, dehydrated, and embedded in paraffin. Sections $(5 \mu \mathrm{m})$ were stained with HE.

\section{Immunohistochemical examinations of the thyroid glands and orbits}

Both the thyroid sections $(5 \mu \mathrm{m})$ and orbital sections $(5 \mu \mathrm{m})$ were incubated with CD3 antibodies (Servicebio, Wuhan, China). In addition, orbital sections were incubated with interferon-gamma (IFN- $\gamma$ ) (Huaan, Hangzhou, China), interleukin (IL)-4 (Huaan), Foxp3 (Servicebio), and IL-17a (Servicebio) antibodies. Images were captured, and the numbers of IFN- $\gamma+$ and IL-4+ cells were counted in three random nonreplicated $400 \times$ fields of each orbit and reported as a ratio (6). The numbers of Foxp3+ and IL-17a+ cells in orbits were similarly enumerated.

\section{Flow cytometry analysis}

Spleen lymphocytes were suspended at a concentration of $1 \times 10^{7}$ cells $/ \mathrm{mL}$ and then stained with FITC-CD4 antibodies (eBioscience, San Diego, CA, USA). Foxp3 expression in CD4+ T cells was detected using a mouse regulatory $\mathrm{T}$ (Treg) cell staining kit according to the manufacturer's protocol (eBioscience). CD4+IL-4+, CD4+IFN- $\gamma+$, and CD4+IL$17 \mathrm{a}+$ cells were stimulated and detected as previously described $(8,21)$. A four-color flow cytometry analysis was performed (FACSAria; BD Biosciences, San Jose, CA, USA), and data were analyzed using FlowJo 7.6.1 software (BD Biosciences).

\section{RNA sequencing analysis}

RNA was isolated from splenocytes and then sequenced after reverse transcription-PCR. Differentially expressed genes (DEGs) were identified through fold change filtering $(p<0.05)$, as described previously (21). A pathway analysis was performed using Gene Set Enrichment Analysis (GSEA) in the $\mathrm{R}$ program (package msigbd, C5) to further explore differences.

\section{Statistical analyses}

All data are presented as means and standard deviations (SD). Differences between groups were analyzed using ANOVA or the independent samples $t$-test. All statistical analyses were performed using SPSS (SPSS 19.0), and $p<0.05$ was considered significant.

\section{Results}

\section{Orbital histopathology of GO mice}

Histopathology and thyroid function were analyzed at predetermined time points (11th, 23rd, and 34th week, namely after four, seven, and nine injections) to thoroughly assess the progression of GO in the mouse model (Fig. 1A). After nine injections, the volume of retrobulbar fibrotic tissue in the AdTSHRA-challenged group was markedly increased compared with the corresponding volumes in the Ad-EGFP-challenged group ( $p=0.044$ ) [Fig. 1B(b)]. The area of retrobulbar adipose tissue was also increased in the Ad-TSHRA-challenged group ( $p=0.022)$ (Fig. 1C). Using the mean \pm 2 SD in the AdEGFP-challenged group as the normal range, 7 of 10 mice (12 of 20 orbits) exhibited retrobulbar fibrosis, and 4 of 10 mice ( 6 of 20 orbits) exhibited retrobulbar adipogenesis in the Ad-TSHRA-challenged group [Fig. 1B(c), C(c) and Table 1]. In addition, one mouse developed exophthalmia and conjunctiva redness, and another mouse developed eyelid hyperplasia and thickening (Fig. 1D). T lymphocyte infiltration in the orbital tissue was also evident in GO mice (Fig. 1E). In summary, the frequency of GO in the Ad-TSHRA-challenged group was $70 \%$ (7 of 10 mice) at the end of the study (Table 1). Retrobulbar fibrosis and adipose deposition were first detected after seven injections (23rd week) (Fig. 1B(c), $\mathrm{C}(\mathrm{c})$ and Table 1).

\section{Hyperthyroidism in mice immunized with Ad-TSHRA}

During the experiment, the daily food intake normalized to body weight was higher in the Ad-TSHRA-challenged group than in the Ad-EGFP-challenged group (Fig. 2A). Persistent hyperthyroidism was confirmed by measuring serum TRAbs and TT4 levels. At all three time points, TRAb levels were elevated in all the Ad-TSHRA-challenged mice, whereas for the Ad-EGFP-challenged mice, TRAbs could not be detected (Fig. 2B and Table 1). After setting the mean $\pm 2 \mathrm{SD}$ of the TT4 levels in the Ad-EGFP-challenged group as the normal range, TT4 levels were increased in most of the Ad-TSHRAchallenged mice $(67 \%, 50 \%$, and $80 \%$ after four, seven, and nine injections, respectively) (Fig. 2C and Table 1).

\section{Histopathological changes in the thyroid glands}

Thyroid glands from all mice were harvested and stained with HE (Fig. 2D). Unlike the Ad-EGFP-challenged mice, hyperplastic changes in the thyroid could be observed in the Ad-TSHRA-challenged mice at all three time points (after four, seven, and nine injections). In particular, thyroid follicular cells exhibited a cuboidal or tall columnar morphology and had papillary folds and protrusions into the follicular cavity.

Immunohistochemistry for CD3 was also performed in the thyroid glands (Fig. 2D). No T lymphocyte infiltration was observed after four injections (at the 11th week) in our study or in the adenovirus-induced GD mouse model reported in previous studies $(7,9)$. However, T lymphocyte infiltration was observed in the thyroid gland after seven and nine injections (at the 23rd and 34th week, respectively).

\section{Changes in CD4+ T cell subsets during immunization}

The relationship between $\mathrm{T}$ cells and autoimmune diseases has been extensively studied (18,22-27). Several subsets of T helper (Th) cells were analyzed to further explore the changes in the immune status during the experiment. Among human peripheral blood cells, CD4+IFN- $\gamma+$ T cells secrete IFN $-\gamma$, which is a representative Th1-type cytokine, and CD4+IL-4+ $\mathrm{T}$ cells secrete IL-4, which is a representative Th2-type cytokine (28). Foxp3 is a specific transcription factor in Treg cells that play an important role in regulating development and function (29). Therefore, in this study, IFN- $\gamma+$, IL-4+, and Foxp3+CD4+ T cells were chosen to reflect Th1, Th2, and Treg cell functions, respectively. 
Table 1. Serological and Morphological Changes in the Adenovirus Thyrotropin Receptor A Subunit-Challenged Mice

\begin{tabular}{|c|c|c|c|c|c|c|c|c|c|c|}
\hline \multirow[b]{4}{*}{ Week } & \multirow[b]{4}{*}{ Injections } & \multirow{4}{*}{$\begin{array}{c}\text { Mouse } \\
\text { no. }\end{array}$} & \multicolumn{2}{|c|}{ Serum } & \multicolumn{5}{|c|}{ Pathogenesis } & \multirow[b]{4}{*}{ Diagnosis $^{\mathrm{e}}$} \\
\hline & & & \multirow{3}{*}{$\begin{array}{c}\text { TRAbs } \\
\text { (positive }^{\mathrm{p}} \\
>5.443 \mathrm{IU} / \mathrm{L})^{\mathrm{a}}\end{array}$} & \multirow{3}{*}{$\begin{array}{c}\text { TT4 } \\
\text { (positive } \\
>37.773 \mu g / d L)^{\mathrm{b}}\end{array}$} & \multirow[b]{3}{*}{ Thyroid $^{\mathrm{c}}$} & \multicolumn{4}{|c|}{ Retrobulbar ${ }^{\mathrm{d}}$} & \\
\hline & & & & & & \multicolumn{2}{|c|}{ Fibrosis } & \multicolumn{2}{|c|}{ Adipogenesis } & \\
\hline & & & & & & $L$ & $R$ & $L$ & $R$ & \\
\hline 11 & 4 & 1 & 43.684 & 28.128 & + & l & l & l & / & NO \\
\hline 11 & 4 & 10 & 75.728 & 57.431 & + & / & / & / & / & $\mathrm{GH}$ \\
\hline 11 & 4 & 14 & 47.346 & 93.351 & + & $\mathrm{N}$ & $\mathrm{N}$ & $\mathrm{N}$ & $\mathrm{N}$ & $\mathrm{GH}$ \\
\hline 11 & 4 & 35 & 37.769 & 101.625 & + & $\mathrm{N}$ & $\mathrm{N}$ & / & / & $\mathrm{GH}$ \\
\hline 11 & 4 & 38 & 21.556 & 25.516 & - & $\mathrm{N}$ & $\mathrm{N}$ & $\mathrm{N}$ & $\mathrm{N}$ & NO \\
\hline 11 & 4 & 39 & 54.525 & 138.732 & + & $\mathrm{N}$ & $\mathrm{N}$ & $\mathrm{N}$ & $\mathrm{N}$ & $\mathrm{GH}$ \\
\hline 23 & 7 & 8 & 25.096 & I & + & I & I & I & I & ND \\
\hline 23 & 7 & 12 & 29.573 & 53.229 & + & I & I & I & / & $\mathrm{GH}$ \\
\hline 23 & 7 & 18 & / & / & + & $\mathrm{N}$ & $\mathrm{N}$ & / & / & ND \\
\hline 23 & 7 & 33 & 65.658 & 41.645 & + & $\mathrm{N}$ & + & $\mathrm{N}$ & $\mathrm{N}$ & $\mathrm{GO}$ \\
\hline 23 & 7 & 41 & 82.254 & 186.912 & + & $\mathrm{N}$ & $\mathrm{N}$ & $\mathrm{N}$ & $\mathrm{N}$ & $\mathrm{GH}$ \\
\hline 23 & 7 & 46 & 25.186 & 26.772 & - & + & $\mathrm{N}$ & $\mathrm{N}$ & $\mathrm{N}$ & $\mathrm{GO}$ \\
\hline 34 & 9 & 13 & 28.417 & 37.855 & + & + & + & I & I & $\mathrm{GO}$ \\
\hline 34 & 9 & 15 & 44.315 & 48.359 & + & I & I & I & I & $\mathrm{GH}$ \\
\hline 34 & 9 & 16 & 60.621 & 52.567 & + & / & / & I & I & $\mathrm{GH}$ \\
\hline 34 & 9 & 31 & 29.891 & 76.006 & + & + & + & I & I & $\mathrm{GO}$ \\
\hline 34 & 9 & 32 & 24.924 & 22.991 & - & + & $\mathrm{N}$ & / & / & $\mathrm{GO}$ \\
\hline 34 & 9 & 34 & 105.355 & 41.275 & + & + & + & + & + & $\mathrm{GO}$ \\
\hline 34 & 9 & 42 & 49.180 & 39.691 & + & $\mathrm{N}$ & + & $\mathrm{N}$ & + & $\mathrm{GO}$ \\
\hline 34 & 9 & 43 & 149.032 & 36.691 & - & $\mathrm{N}$ & $\mathrm{N}$ & / & I & NO \\
\hline 34 & 9 & 44 & 29.069 & 44.986 & - & + & + & + & + & GO \\
\hline 34 & 9 & 45 & 12.333 & 45.549 & + & + & + & + & $\mathrm{N}$ & GO \\
\hline
\end{tabular}

A total of nine mice diagnosed with GO (seven after nine injections and two after seven injections), and all of them showed positive TRAbs. Among them, two mice showed negative TT4 (Nos. 32 and 46) and four mice suffered unilateral ocular changes (Nos. 33, 46, 32, and 42).

${ }^{a}$ TRAbs: Ad-EGFP-challenged mice, mean $\pm 2 \mathrm{SD}=5.443 \mathrm{IU} / \mathrm{L}$. Higher values indicated significant positivity.

${ }^{\mathrm{b}}$ TT4: Ad-EGFP-challenged mice, mean $\pm 2 \mathrm{SD}=37.773 \mu \mathrm{g} / \mathrm{dL}$. Higher values (bold) indicated hyperthyroidism.

${ }^{c}$ Pathogenesis of thyroid glands: -, normal thyroid morphology; +, proliferation and hypertrophy of follicular cells were observed (Fig. 2D).

${ }^{d}$ Pathogenesis of orbits: + , higher than mean \pm 2 SD of the Ad-EGFP-challenged mice; N, normal.

${ }^{\mathrm{e}}$ Diagnosis: GO, existing orbital pathogenesis; GH, elevated TT4 without evidence of existing orbital pathogenesis; NO, normal TT4 without evidence of existing orbital pathogenesis; ND, no diagnosis.

I, not determined; Ad-EGFP, adenovirus expressing green fluorescent protein; GH, Graves' hyperthyroidism; GO, Graves' orbitopathy; $\mathrm{SD}$, standard deviation; TRAb, serum autoantibody; TT4, total thyroxine.

The percentage of Th1 cells was significantly increased $(p=0.024)$, and the percentage of Th2 cells was significantly decreased $(p<0.001)$ in the Ad-TSHRA-challenged mice after nine injections (at the 34th week) compared with the Ad-EGFP-challenged mice (Fig. 3B). A noticeably lower proportion of Treg cells was detected in the Ad-TSHRAchallenged group than in the Ad-EGFP-challenged group $(p=0.001)$ (Fig. 3A). However, the Th17 proportion in the two groups was not significantly different (Fig. 3B), which was most likely related to the genetic background of the mice (30). In addition, the proportion of Th2 cells was lower after nine injections (at the 34th week) than after four injections (at the 11th week) $(p=0.022)$. No other differences were observed among the three time points.

The Th1 dominance in the Th1/Th2 balance and Treg cell elimination were confirmed by orbital immunohistochemical staining. After nine injections, the Ad-TSHRA-challenged mice displayed a significantly decreased number of IL-4+ and Foxp3+ cells in the orbits compared with the AdEGFP-challenged mice ( $p=0.001$ and $p<0.001$, respectively)
(Fig. 3C, D). Noticeably, IFN- $\gamma+$ cells had infiltrated in the two orbits of the Ad-TSHRA-challenged mice (Fig. 3C). Although the number of IFN- $\gamma+$ cells was not significantly different in the two groups, the ratio of IFN- $\gamma+$ :IL- $4+$ cells was significantly increased in the Ad-TSHRA-challenged mice $(p=0.042)$. No IL17a+ cells were observed in the orbits of both groups.

\section{DEGs and pathways in RNA sequencing analysis}

RNA sequencing was performed in nine mice: three mice challenged with Ad-EGFP, three mice that received three injections of Ad-TSHRA (at the 11th week) and were diagnosed with GH (Nos. 14, 35, and 39), and three mice that received nine injections of Ad-TSHRA (at the 34th week) and were diagnosed with GO (Nos. 34, 42, and 44).

Compared with the mice challenged with Ad-EGFP, 1061 downregulated genes and 160 upregulated genes were identified in the gene expression profile of GO mice (at the 34th week) (Fig. 4A). An analysis of gene ontology terms revealed 

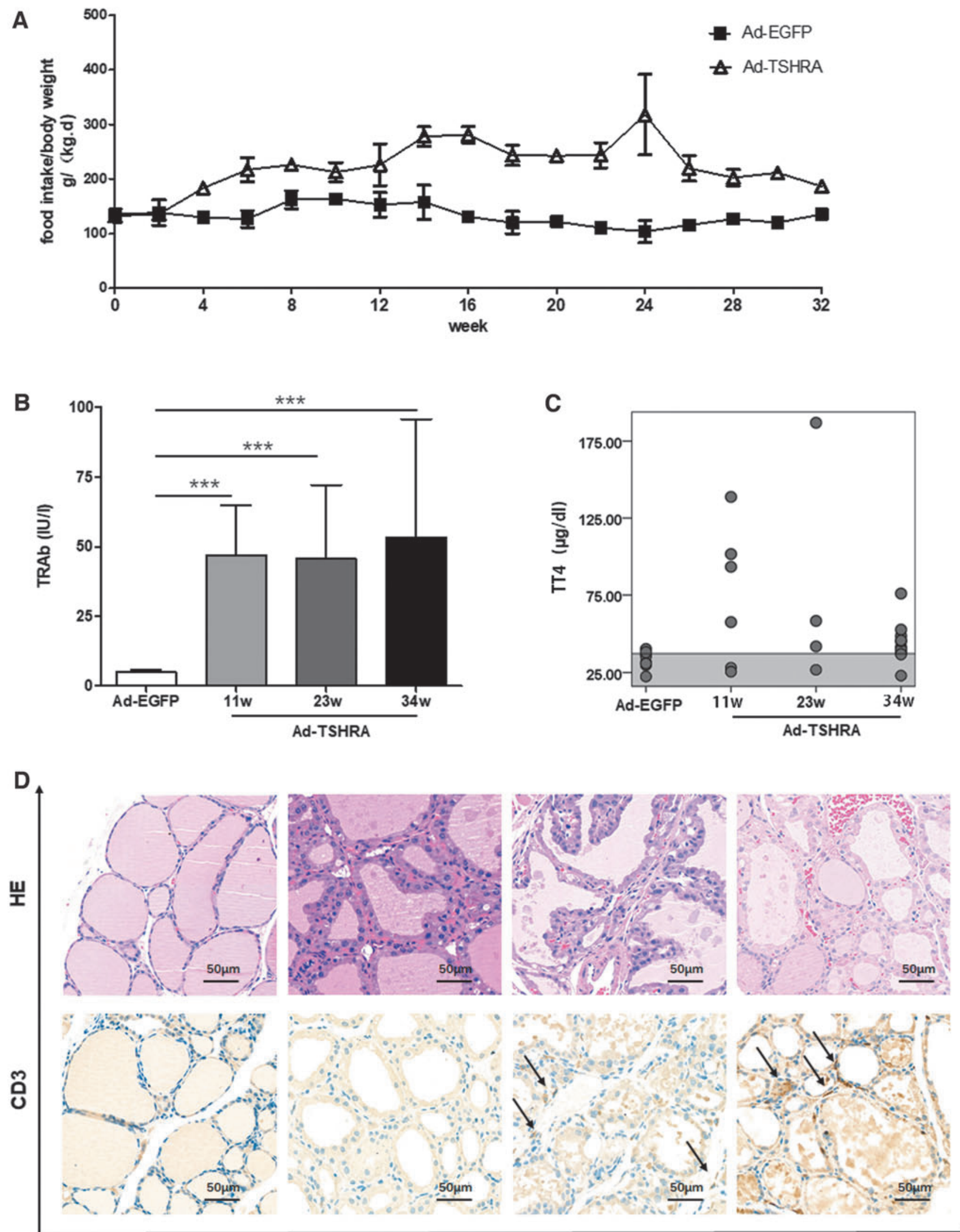

Ad-EGFP

Ad-TSHRA 11w

Ad-TSHRA 23w

Ad-TSHRA 34w

FIG. 2. (A) Daily food intake normalized to body weight during the entire experiment. (B) TRAb levels of the Ad-EGFPand Ad-TSHRA-challenged mice at different times. (C) Using the mean \pm 2 SD TT4 level of the Ad-EGFP-challenged mice as the normal range, most mice challenged with Ad-TSHRA had high TT4 levels (4/6, 3/4, and 8/10 in weeks 11, 23, and 34, respectively). (D) Thyroid pathology of the Ad-EGFP- and Ad-TSHRA-challenged mice at different times $(\times 40)$. Infiltrating lymphocytes are indicated by arrows. $* * * p<0.001$. TRAb, serum autoantibody; TT4, total thyroxine. 

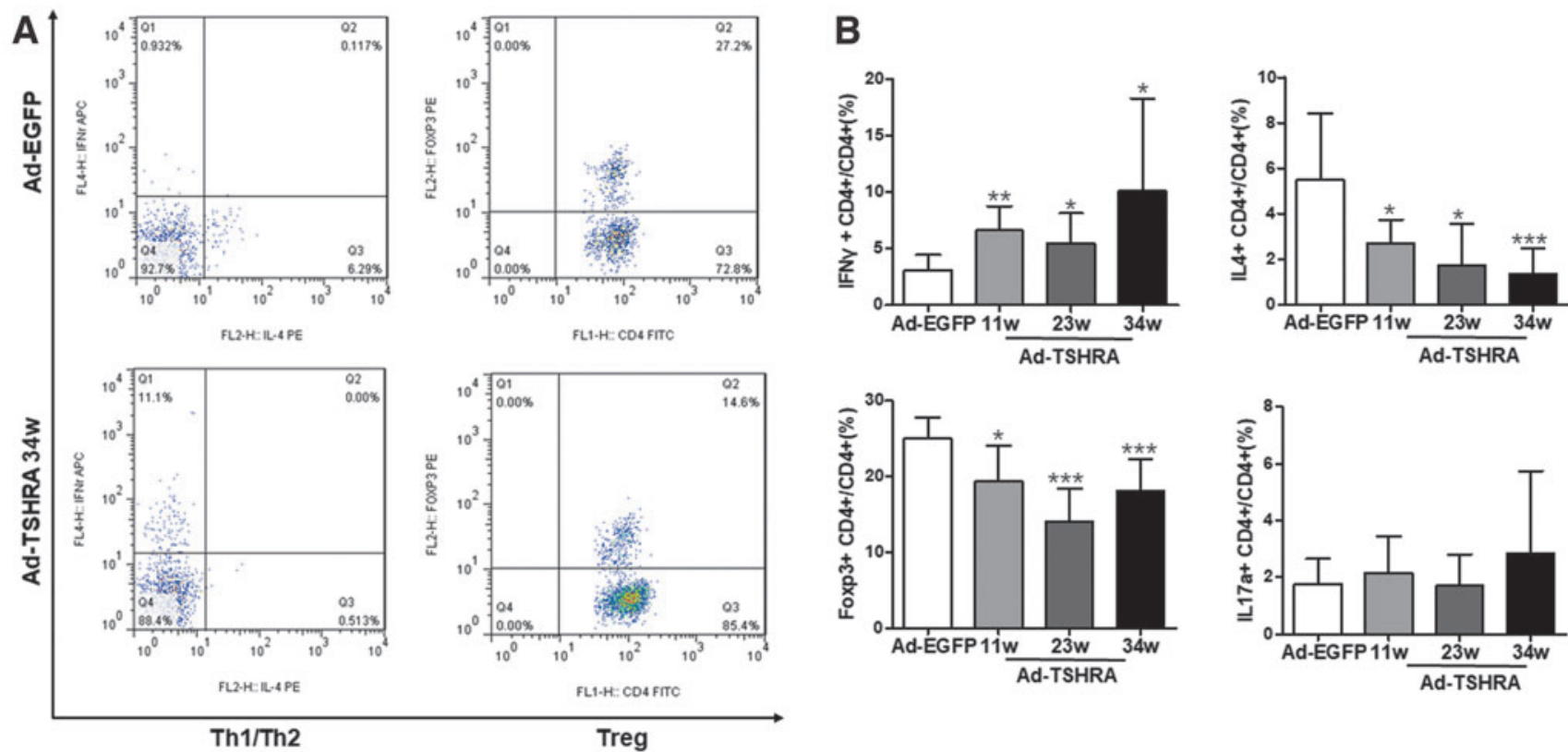

Th1/Th2

Treg
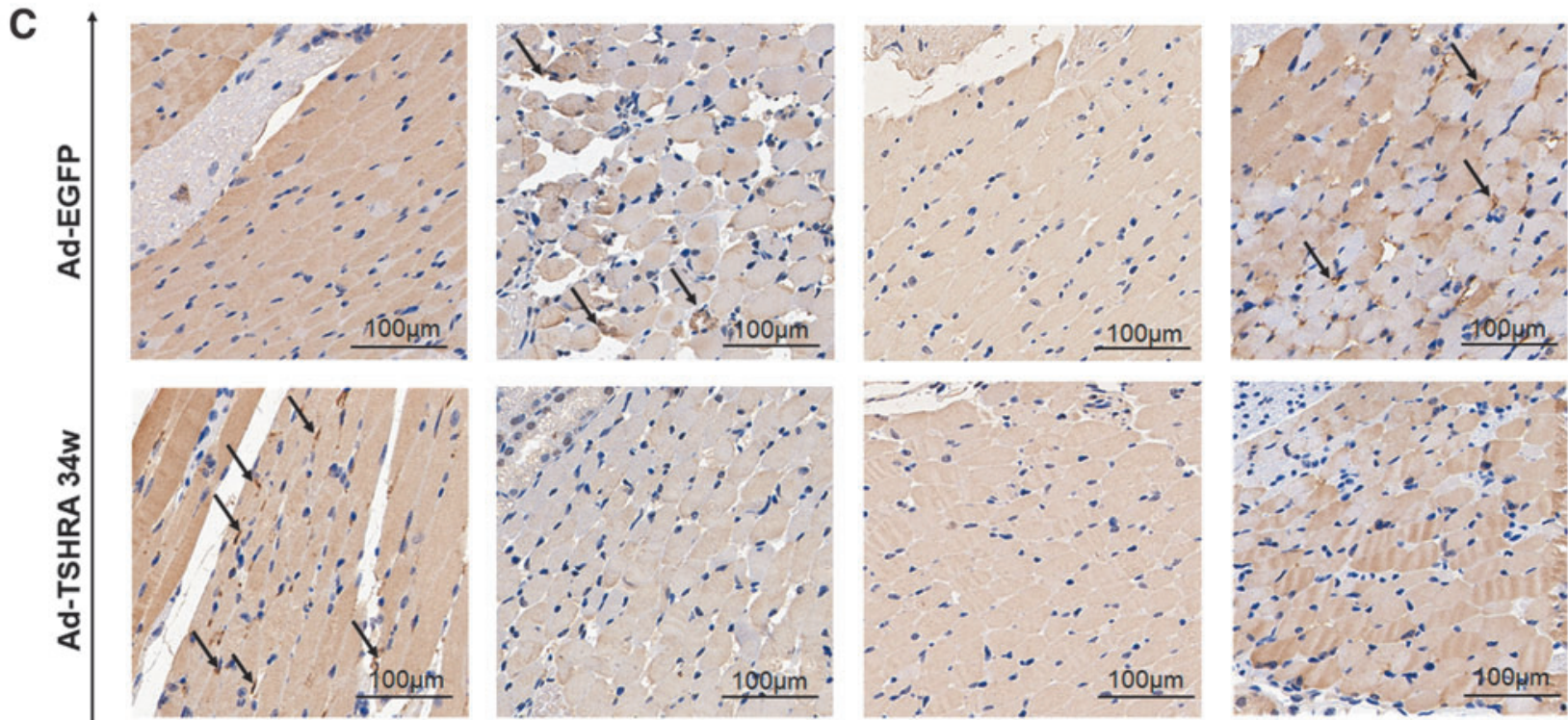

IFN-r
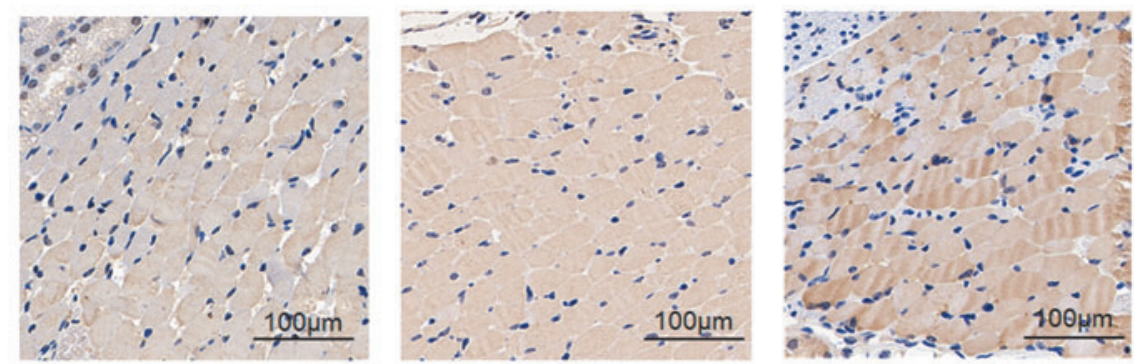

$\mathrm{IL}-4$

IL-17

Foxp3

D
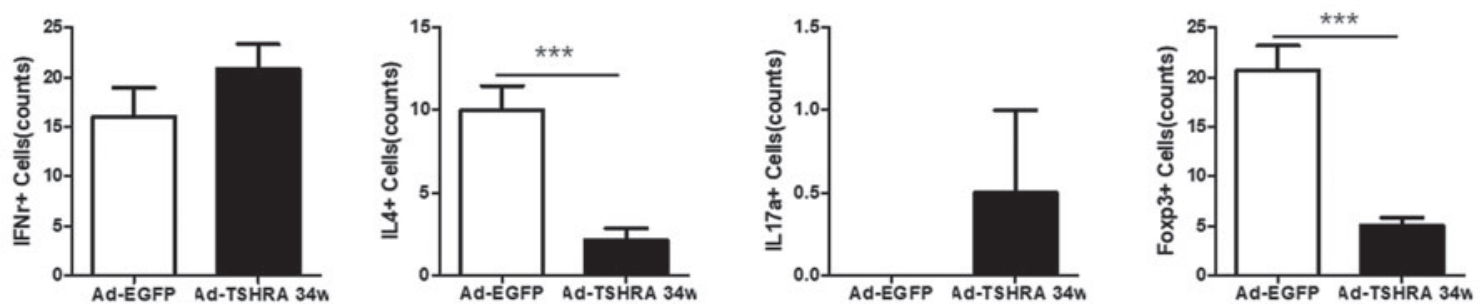

FIG. 3. (A) Flow cytometric analysis of Th1 and Th2 cells and Tregs of the Ad-EGFP- and Ad-TSHRA-challenged mice at week 34. (B) Ratios of Th1, Th2, and Th17 cells and Tregs of the Ad-EGFP- and Ad-TSHRA-challenged mice at different times. (C) Orbital immunohistochemical results of the Ad-EGFP- and Ad-TSHRA-challenged mice at week 34. The positive cells are shown in black arrows. (D) Numbers of interferon-gamma+, interleukin-4+, IL-17a+, and Foxp3+ cells in orbits of the Ad-EGFP- and Ad-TSHRA-challenged mice. ${ }^{*} p<0.05,{ }^{*} p<0.01, * * * p<0.001$. 


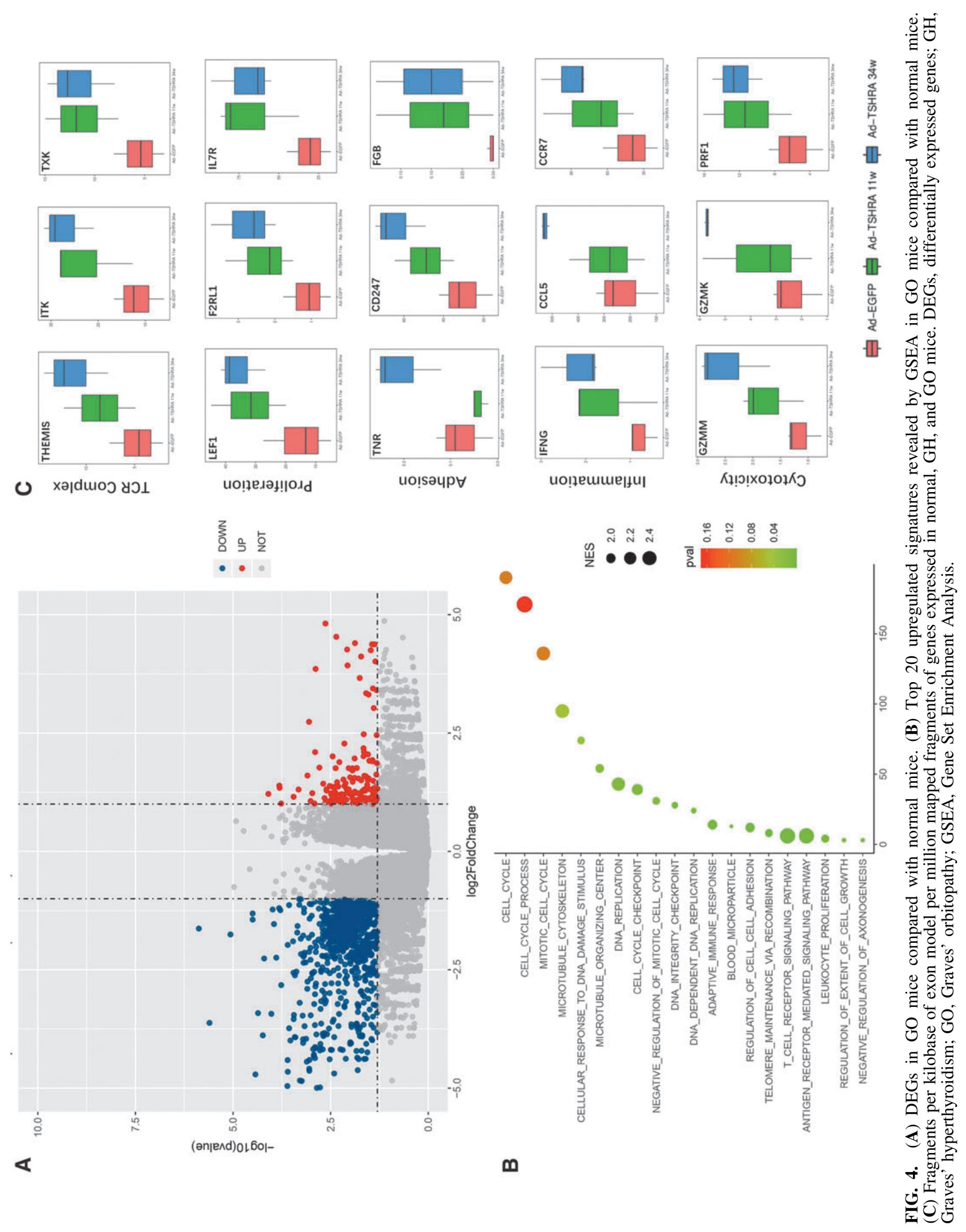


that the top 20 upregulated signatures in the GO mice included the T cell receptor (TCR) pathway, leukocyte proliferation, and regulation of cell-cell adhesion (Fig. 4B). Moreover, terms related to positive regulation of the acute inflammatory response $(p=0.035$, normalized enrichment score $[\mathrm{NES}]=1.537)$ and regulation of cell killing $(p=0.011$, $\mathrm{NES}=1.823)$ were also upregulated in the GO mice.

A more detailed analysis confirmed that spleen cells expressed TCR complex-, proliferation-, inflammation-, and cytotoxicity-related genes at significantly higher levels in GO mice than in mice challenged with Ad-EGFP (Fig. 4C). Furthermore, a tendency toward upregulation of genes, particularly inflammation- and cytotoxicity-related genes (IFNG, CCL5, CCR7, GZMM, and GZMK), in the GH mice (after four injections) was observed, although it was not statistically significant (Fig. 4C). Based on these findings, changes in the $\mathrm{T}$ cell immunity associated with GO evolved in mice with hyperthyroidism without orbitopathy.

\section{Discussion}

In the present study, we established a mouse model of GO with a high frequency $(70 \%)$ by administering nine injections of Ad-TSHRA. The progression from $\mathrm{GH}$ to $\mathrm{GO}$ was also observed and highlighted by a series of changes in $\mathrm{T}$ cell immunity.

After prolonged immunizations with Ad-TSHRA, retrobulbar adipogenesis, lymphocyte infiltration, and several clinical signs, in addition to the previously reported fibrosis, were observed for the first time in this long-term animal model (14-17). The frequency of the orbital changes was $70 \%$ and was consistent with the incidence of subclinical eye involvement $(2,31)$. Hyperthyroidism was also observed and was manifested as positive TRAbs, elevated TT4 levels, and hyperplastic thyroid morphology compared with the most orbitopathy models reported in previous studies $(6,19,32)$. In summary, this model recapitulated most of the characteristics of an ideal GO model (32).

Both GO and GH mainly depend on T cells $(2,18)$. In our GO model, CD3+ lymphocytes were observed in both the thyroid gland and the orbit. The Th1 dominance in the Th1/ Th2 cell balance and Treg cell elimination were present both in the spleen and in the orbit (24,33-38). Moreover, in splenocytes of GO mice, the expression of genes involved in pathways related to the TCR complex, proliferation, adhesion, inflammation, and cytotoxicity was upregulated. Cytokines secreted by Th1 cells regulate the recruitment of inflammatory cells and subsequently promoting orbital remodeling (2). Additionally, the increased activity of the TCR complex may also be related to positive TRAbs from B cells, due to its importance in antigen recognition $(2,39)$. Thus, we postulated that $\mathrm{T}$ cell immunity was a critical component of the pathogenesis of GO, particularly in initiating and perpetuating the autoimmune-inflammation cascade.

The progression from $\mathrm{GH}$ to $\mathrm{GO}$ was reproduced in the model, and the GO-specific T cell immunity observed during the progression should be of particular concern. Hyperthyroidism, similar to other reported studies $(7,9)$, was observed after four injections, whereas orbitopathy was identified after seven injections. CD3+ T lymphocyte infiltration and an imbalance of CD4+ Th cells in the orbits also followed these changes in the thyroid or the spleen. In addition to positive
TRAbs (19), the functional changes in T cell immunity described above are responsible for the onset of GO, which already existed in the splenocytes of mice with hyperthyroidism after four injections. Thus, a time window for the progression from $\mathrm{GH}$ to $\mathrm{GO}$ (after four injections) corresponding to clinical findings was identified in our model (18); this time window might be defined as a "latent phase" of GO, during which GO-specific $\mathrm{T}$ cell immunity evolves in mice with hyperthyroidism before ophthalmopathy occurs.

Although much of the orbital changes coexisted with hyperthyroidism, two mice only exhibited orbital lesions (Ad-TSHRA-injected mouse Nos. 32 and 46) (Table 1). This phenomenon is consistent with a few cases called euthyroid Graves' ophthalmopathy (EGO), which is defined as the presence of thyroid-associated ophthalmopathy in patients with normal thyroid function $(40,41)$. Most of the patients with EGO proved to be TRAb-positive (including stimulating and inhibiting antibodies), but the underlying mechanism is currently unknown (41). Only a few antibodies generated in BALB/c mice immunized with human TSHRA cross-react with the mouse TSHR to induce GH, likely due to the heterogeneity of TSHR between humans and mice (42). Therefore, further studies are needed to clarify the mechanism.

There are several limitations of this study. First, both stimulating and blocking antibodies coexist in the serum of mice immunized with the TSHR cDNA $(10,11,13)$; however, only the total levels of TRAbs were measured in this study due to technical limitations. We suggest that the detected antibodies were predominantly stimulating antibodies because of the elevated TT4 levels. Second, we did not perform orbital 7-T magnetic resonance imaging to observe changes in extraocular muscles, which should be conducted in future studies. Finally, the area of adipose tissue was not calculated in all mice because of technical limitations.

Taken together, a murine model of GO was successfully induced by immunizations with Ad-TSHRA, with a high frequency $(70 \%)$. The important role of T cells in the pathogenesis of GO was characterized, and the progression from $\mathrm{GH}$ to $\mathrm{GO}$ was reproduced for the first time in our study. This model will be a useful tool for further pathogenesis studies and therapeutic management of GO.

\section{Author Disclosure Statement}

No competing financial interests exist.

\section{Funding Information}

This study was supported by the National Key R\&D Program of China [Grant No. 2018YFC1311500 (B.-Y.S.)], the National Science Foundation of China (NSFC) [Grant Nos. 81500690 (Y.W.) and 81970679 (B.-Y.S.)], the Natural Science Foundation of Shaanxi Province [Grant No. 2018JM70990 (Y.W.)], the Key Research and Development Project of Shaanxi Province [Grant No. 2017ZDXM-SF-060 (B.-Y.S.)], the Fundamental Research Funds for the Central Universities [Grant No. 1191329875 (Y.W.)], and the China Postdoctoral Science Foundation [Grant No. 224646 (Y.W.)].

\section{References}

1. Smith TJ, Hegedüs L 2016 Graves' disease. N Engl J Med 375:1552-1565. 
2. Bahn RS 2010 Graves' ophthalmopathy. N Engl J Med 362:726-738.

3. Lazarus JH 2012 Epidemiology of Graves' orbitopathy (GO) and relationship with thyroid disease. Best Pract Res Clin Endocrinol Metab 26:273-279.

4. Piantanida E, Tanda ML, Lai A, Sassi L, Bartalena L 2013 Prevalence and natural history of Graves' orbitopathy in the XXI century. J Endocrinol Invest 36:444-449.

5. Menconi F, Leo M, Sabini E, Mautone T, Nardi M, Sainato A, Sellari-Franceschini S, Vitti P, Marcocci C, Marinò M 2017 Natural history of graves' orbitopathy after treatment. Endocrine 57:226-233.

6. Many MC, Costagliola S, Detrait M, Denef F, Vassart G, Ludgate MC 1999 Development of an animal model of autoimmune thyroid eye disease. J Immunol 162:49664974.

7. McLachlan SM, Nagayama Y, Rapoport B 2005 Insight into Graves' hyperthyroidism from animal models. Endocr Rev 26:800-832.

8. Wang Y, Wu LP, Fu J, Lv HJ, Guan XY, Xu L, Chen P, Gao CQ, Hou P, Ji MJ, Shi BY 2013 Hyperthyroid monkeys: a nonhuman primate model of experimental Graves' disease. J Endocrinol 219:183-193.

9. Nagayama Y 2005 Animal models of Graves' hyperthyroidism. Endocr J 52:385-394.

10. Zhao S-X, Tsui S, Cheung A, Douglas RS, Smith TJ, Banga JP 2011 Orbital fibrosis in a mouse model of Graves' disease induced by genetic immunization of thyrotropin receptor cDNA. J Endocrinol 210:369-377.

11. Moshkelgosha S, So P-W, Deasy N, Diaz-Cano S, Banga JP 2013 Cutting edge: retrobulbar inflammation, adipogenesis, and acute orbital congestion in a preclinical female mouse model of Graves' orbitopathy induced by thyrotropin receptor plasmid-in vivo electroporation. Endocrinology 154:3008-3015.

12. Xia N, Ye X, Hu X, Song S, Xu H, Niu M, Wang H, Wang J 2017 Simultaneous induction of Graves' hyperthyroidism and Graves' ophthalmopathy by TSHR genetic immunization in BALB/c mice. PLoS ONE 12:e0174260.

13. Berchner-Pfannschmidt U, Moshkelgosha S, Diaz-Cano S, Edelmann B, Görtz G-E, Horstmann M, Noble A, Hansen W, Eckstein A, Banga JP 2016 Comparative assessment of female mouse model of Graves' orbitopathy under different environments, accompanied by proinflammatory cytokine and T-cell responses to thyrotropin hormone receptor antigen. Endocrinology 157:1673-1682.

14. Holthoff H-P, Goebel S, Li Z, Faßbender J, Reimann A, Zeibig S, Lohse MJ, Münch G, Ungerer M 2015 Prolonged TSH receptor A subunit immunization of female mice leads to a long-term model of Graves' disease, tachycardia, and cardiac hypertrophy. Endocrinology 156:1577-1589.

15. Holthoff H-P, Li Z, Faßbender J, Reimann A, Adler K, Münch G, Ungerer M 2017 Cyclic peptides for effective treatment in a long-term model of Graves disease and orbitopathy in female mice. Endocrinology 158:2376-2390.

16. Ungerer M, Faßbender J, Li Z, Münch G, Holthoff H-P 2017 Review of mouse models of Graves' disease and orbitopathy-novel treatment by induction of tolerance. Clin Rev Allergy Immunol 52:182-193.

17. Faßbender J, Holthoff H-P, Li Z, Ungerer M 2019 Therapeutic effects of short cyclic and combined epitope peptides in a long-term model of Graves' disease and orbitopathy. Thyroid 29:258-267.
18. McLachlan SM, Prummel MF, Rapoport B 1994 Cellmediated or humoral immunity in Graves' ophthalmopathy? Profiles of T-cell cytokines amplified by polymerase chain reaction from orbital tissue. J Clin Endocrinol Metab 78:1070-1074.

19. Banga JP, Moshkelgosha S, Berchner-Pfannschmidt U, Eckstein A 2015 Modeling Graves' orbitopathy in experimental Graves' disease. Horm Metab Res 47:797-803.

20. Dahab GM, Kheriza MM, El-Beltagi HM, Fouda A-MM, El-Din OAS 2004 Digital quantification of fibrosis in liver biopsy sections: description of a new method by Photoshop software. J Gastroenterol Hepatol 19:78-85.

21. Wang Y, Zhao F, Rijntjes E, Wu L, Wu Q, Sui J, Liu Y, Zhang M, He M, Chen P, Hu S, Hou P, Schomburg L, Shi B 2019 Role of selenium intake for risk and development of hyperthyroidism. J Clin Endocrinol Metab 104:568-580.

22. Wu G, Wu N, Li T, Lu W, Yu G 2016 Total glucosides of peony ameliorates Sjögren's syndrome by affecting Th1/Th2 cytokine balance. Exp Ther Med 11:1135-1141.

23. Zhang S, Liu X, Sun C, Yang J, Wang L, Liu J, Gong L, Jing Y 2016 Apigenin attenuates experimental autoimmune myocarditis by modulating Th1/Th2 cytokine balance in mice. Inflammation 39:678-686.

24. Huang Y, Fang S, Li D, Zhou H, Li B, Fan X 2019 The involvement of $\mathrm{T}$ cell pathogenesis in thyroid-associated ophthalmopathy. Eye (Lond) 33:176-182.

25. Wakelkamp IMMJ, Bakker O, Baldeschi L, Wiersinga WM, Prummel MF 2003 TSH-R expression and cytokine profile in orbital tissue of active vs. inactive Graves' ophthalmopathy patients. Clin Endocrinol (Oxf) 58:280-287.

26. Kim SE, Yoon JS, Kim KH, Lee SY 2012 Increased serum interleukin-17 in Graves' ophthalmopathy. Graefes Arch Clin Exp Ophthalmol 250:1521-1526.

27. Fang S, Huang Y, Wang S, Zhang Y, Luo X, Liu L, Zhong S, Liu X, Li D, Liang R, Miranda P, Gu P, Zhou H, Fan X, Li B 2016 IL-17A exacerbates fibrosis by promoting the proinflammatory and profibrotic function of orbital fibroblasts in TAO. J Clin Endocrinol Metab 101:29552965.

28. Mosmann TR, Coffman RL 1989 TH1 and TH2 cells: different patterns of lymphokine secretion lead to different functional properties. Annu Rev Immunol 7:145-173.

29. Nie J, Li YY, Zheng SG, Tsun A, Li B 2015 FOXP3(+) Treg cells and gender bias in autoimmune diseases. Front Immunol 6:493.

30. Horie I, Abiru N, Saitoh O, Ichikawa T, Iwakura Y, Eguchi K, Nagayama Y 2011 Distinct role of T helper Type 17 immune response for Graves' hyperthyroidism in mice with different genetic backgrounds. Autoimmunity 44:159-165.

31. Enzmann DR, Donaldson SS, Kriss JP 1979 Appearance of Graves' disease on orbital computed tomography. J Comput Assist Tomogr 3:815-819.

32. Ludgate M, Baker G 2004 Inducing Graves' ophthalmopathy. J Endocrinol Invest 27:211-215.

33. Hiromatsu Y, Yang D, Bednarczuk T, Miyake I, Nonaka K, Inoue Y 2000 Cytokine profiles in eye muscle tissue and orbital fat tissue from patients with thyroid-associated ophthalmopathy. J Clin Endocrinol Metab 85:1194-1199.

34. Han R, Smith TJ 2006 T helper type 1 and type 2 cytokines exert divergent influence on the induction of prostaglandin E2 and hyaluronan synthesis by interleukin-1beta in orbital fibroblasts: implications for the pathogenesis of thyroidassociated ophthalmopathy. Endocrinology 147:13-19. 
35. Khanna D, Chong KKL, Afifiyan NF, Hwang CJ, Lee DK, Garneau HC, Goldberg RA, Darwin CH, Smith TJ, Douglas RS 2010 Rituximab treatment of patients with severe, corticosteroid-resistant thyroid-associated ophthalmopathy. Ophthalmology 117:133.

36. Matsuzawa K, Izawa S, Okura T, Fujii S, Matsumoto K, Shoji K, Nakamura R, Sumi K, Fujioka Y, Yoshida A, Shigemasa C, Kato M, Yamamoto K, Taniguchi S-I 2016 Implications of FoxP3-positive and -negative CD4(+) CD25(+) T cells in Graves' ophthalmopathy. Endocr J 63: 755-764.

37. Xia N, Zhou S, Liang Y, Xiao C, Shen H, Pan H, Deng H, Wang N, Li QQ 2006 CD4+ T cells and the Th1/Th2 imbalance are implicated in the pathogenesis of Graves' ophthalmopathy. Int J Mol Med 17:911-916.

38. Kahaly GJ, Shimony O, Gellman YN, Lytton SD, EshkarSebban L, Rosenblum N, Refaeli E, Kassem S, Ilany J, Naor D 2011 Regulatory T-cells in Graves' orbitopathy: baseline findings and immunomodulation by anti-T lymphocyte globulin. J Clin Endocrinol Metab 96:422-429.

39. Riley TP, Hellman LM, Gee MH, Mendoza JL, Alonso JA, Foley KC, Nishimura MI, Vander Kooi CW, Garcia KC, Baker BM $2018 \mathrm{~T}$ cell receptor cross-reactivity expanded by dramatic peptide-MHC adaptability. Nat Chem Biol 14: 934-942.

40. Termote K, Decallonne B, Mombaerts I 2014 The influence of prior hyperthyroidism on euthyroid graves' ophthalmopathy. J Ophthalmol 2014:426898.

41. Suzuki N, Noh JY, Kameda T, Yoshihara A, Ohye H, Suzuki M, Matsumoto M, Kunii Y, Iwaku K, Watanabe N, Mukasa K, Kozaki A, Inoue T, Sugino K, Ito K 2018
Clinical course of thyroid function and thyroid associatedophthalmopathy in patients with euthyroid Graves' disease. Clin Ophthalmol 12:739-746.

42. Nakahara M, Mitsutake N, Sakamoto H, Chen C-R, Rapoport B, McLachlan SM, Nagayama Y 2010 Enhanced response to mouse thyroid-stimulating hormone (TSH) receptor immunization in TSH receptor-knockout mice. Endocrinology 151:4047-4054.

Address correspondence to: Bing-Yin Shi, MD

The First Affiliated Hospital $X i$ 'an Jiaotong University 277 Yanta West Road $X i$ 'an

Shaanxi Province 710061

China

E-mail: shibingy@126.com

Yue Wang, MD, PhD

The First Affiliated Hospital $X i$ 'an Jiaotong University 277 Yanta West Road $X i$ 'an

Shaanxi Province 710061

China

E-mail: shelly1021@126.com 